\title{
Photocatalytic Activity of Heavy Metal Doped CdS Nanoparticles Synthesized by Using Ocimum sanctum Leaf Extract
}

\author{
Bhanu Mullamuri ${ }^{1(\mathbb{D})}$, Venkata Sai Sriram Mosali ${ }^{1,2}{ }^{(\mathbb{D})}$, Hussen Maseed ${ }^{3(\mathbb{D})}$, Syam Sundar Majety ${ }^{1(\mathbb{D})}$ \\ Basavaiah Chandu ${ }^{1, * \text { (iD }}$ \\ 1 Dept. of Nanotechnology, Acharya Nagarjuna University, Guntur, Andhra Pradesh, India \\ 2 School of Chemistry, Monash University, Clayton - 3800, Victoria Australia \\ 3 School of Engineering Science and Technology, University of Hyderabad, Gachibowli, Hyderabad - 500046, India \\ * Correspondence: chandupcu@gmail.com;
}

Scopus Author ID 57193207705

Received: 10.12.2020; Revised: 20.01.2021; Accepted: 22.01.2021; Published: 30.01.2021

\begin{abstract}
Green chemical approaches for the production of nanomaterials are currently attracted to research and industry development to minimize the use of hazardous chemicals and reduce industrial pollution. A green reagent Ocimum sanctum leaf extract was used to synthesize doped and undoped $\mathrm{CdS}$ nanoparticles in this work. The as-synthesized nanoparticles were characterized by using different characterization techniques such as UV-Vis spectroscopy, XRD, and TEM. The effect of doping on physicochemical properties was analyzed. Photocatalytic properties of these nanoparticles were investigated by sunlight-based photodegradation studies of methylene blue dye. The doping of these green synthesized $\mathrm{CdS}$ nanoparticles with heavy metals has improved photocatalytic efficiency over a given time.
\end{abstract}

Keywords: CdS nanoparticles; doping; photocatalysis; methylene blue.

(C) 2021 by the authors. This article is an open-access article distributed under the terms and conditions of the Creative Commons Attribution (CC BY) license (https://creativecommons.org/licenses/by/4.0/).

\section{Introduction}

The integration of biochemistry with nanotechnology leads to an interdisciplinary platform that enhances the probability of controlling adverse effects of toxicity pervaded in nanomaterials and the environment [1-4]. The unique size-dependent properties of nanoparticles made them the core components in the current century research arena. Even though physical and chemical methods were explored for the effective synthesis of nanomaterials, toxic materials or harmful methods are the major concern for the sustainability of natural habitat and limiting their efficient utilization in various biomedical applications. To overcome these issues, bio-encapsulation or bio-reduction of nanoparticles is adopted $[5,6]$ not just because of reduced utilization of toxic chemicals but also the toxicity of synthesized nanoparticles. Further, the utilization of leaf extracts greatly reduces the use of potentially toxic chemicals, cost of production, pollution, and sustainable development.

On the other hand, organic dyes are being extensively produced and used in textile, paper, cosmetic, pharmaceutical leather, and food industries [7-9]. When released into the environment, the carcinogenic and toxic dyes, along with industrial wastes, would cause severe problems to aquatic life. These dyeing effluents were highly stable to light, heat, and oxidizing agents resulting in weak biodegradability [9]. To circumvent the potential problems of dye 
pollution; nanoparticles were extensively studied as photocatalysts by employing them for the discoloration of these pollutants [7,10-12]. Several semiconductor photocatalysts such as $\mathrm{TiO}_{2}$, $\mathrm{SnO}_{2}, \mathrm{ZnO}, \mathrm{NaTaO}, \mathrm{ZnS} \mathrm{CdS}, \mathrm{CdSe}, \mathrm{Cd}_{1-\mathrm{x}} \mathrm{Zn}_{\mathrm{x}} \mathrm{S}$ have been reported, which showed promising performance in photocatalysis. Among them, CdS NPs are very promising photocatalysts due to their unique optical and photocatalytic properties. A wide forbidden band of $2.42 \mathrm{eV}$ of $\mathrm{CdS}$ NPs is very desirable for photocatalysis driven by visible light. High specific surface area, high crystallinity, short bulk to surface diffusion, and exciton stability are some of the advantages of CdS NPs in visible light-driven photocatalysis. CdS nanoparticles have been widely explored over the past two decades, mainly because of their quantum size effects and photocatalytic properties.

Prominent photocatalytic dye degradation reports on the degradation of photocatalytic dyes have been discussed to emphasize the present study's importance. For instance, Bokare $e t$ al. [13] have synthesized Fe-Ni bimetallic nanoparticles by chemical reduction method. They performed dye degradation studies over Orange $\mathrm{G}$ using the as-synthesized nanoparticles. They reported that with $3 \mathrm{~g} / \mathrm{L}$ catalysts and $150 \mathrm{mg} / \mathrm{L}$ dye loading, the dye was decolorized within 10 min with a rate constant of $0.087 \pm 0.02 \mathrm{~min}^{-1}$. On the other hand, Joseph et al. [14] have prepared Ag nanoparticles using B. sensitivum leaf extract as a capping agent in a microwave oven. The thus prepared samples were reported to exhibit excellent photocatalytic degradation efficiency over methylene blue and methyl orange. Similarly, the Ravikumar group [15] has synthesized $\mathrm{CuO}$ nanoparticles by using Carica papaya leaves extract as a capping agent.

Their photocatalytic studies over Coomassie brilliant blue under sunlight exhibit $\sim 70 \%$ degradation in $2 \mathrm{~h}$. Among the narrow bandgap semiconductors, $\mathrm{CdS}$ is one of the widely used for photocatalytic and antibacterial studies [16,17]. In spite of having a bandgap in the visible region, it suffers from early recombination of electron-hole pairs and weak stability due to photo corrosion [18-20]. Various strategies were implemented to attain a practical solution to improve photocatalytic activity, such as metal ion doping, metal deposition, and compositing with other materials [7, 21-24]. Among these, metal ion doping can improve photocatalytic efficiency as it can alter the bandgap [25-27]. For example, Pouretedal et al. [28,29], synthesized undoped and $\mathrm{Mn}, \mathrm{Ni}$, and $\mathrm{Cu}$ doped $\mathrm{ZnS}$ and $\mathrm{CdS}$ nanoparticles by using controlled precipitation co-precipitation and studied the doping effect on the photocatalytic efficiency of $\mathrm{ZnS}$ and $\mathrm{CdS}$ nanoparticles. They have performed dye degradation studies over methylene blue. Safranin using different doped sulfide nanoparticles with varying doping percentages. They found that $\mathrm{ZnS} / \mathrm{CdS}$ 's catalytic efficiency depends not only on the dopant alone but also on the concentration of the dopants. Thus, the photocatalytic performances of $\mathrm{ZnS}$ catalysts reported were in the order $\mathrm{ZnS}<\mathrm{Zn}_{0.98} \mathrm{Mn}_{0.02} \mathrm{~S}<\mathrm{Zn}_{0.94 \mathrm{Ni} 0.04 \mathrm{~S}}<\mathrm{Zn}_{0.90} \mathrm{Cu}_{0.10} \mathrm{~S}$. Pandey group [30] has performed similar studies over $\mathrm{ZnS}$ nanoparticles, which shows the contrary results. They synthesized $\mathrm{ZnS}$ nanoparticles by chemical precipitation method using thioglycerol as a capping agent and doped them with $\mathrm{Mn}, \mathrm{Ni}$. Their photocatalytic studies over crystal violet have revealed that doping $\mathrm{ZnS}$ with either $\mathrm{Ni}$ or $\mathrm{Mn}$ has an adverse effect. However, their systematic study has revealed that the catalytic performance of nanomaterials is concentrationdependent. Similar studies by Rajabi et al. [31] on chemically prepared Mn, Ni, Co-doped ZnS quantum dots show that on doping, methylene violet's degradation time has reduced considerably compared to undoped ZnS. Chauhan et al. [32], have studied the dye degradation efficiencies of Fe-doped CdS nanoparticles over methylene blue dye. From their studies on doping CdS with different amounts of Fe, the catalyst's bandgap has decreased and hence increased the catalytic efficiency. Recently, the Salam group [33], has synthesized $\mathrm{Pt}, \mathrm{In}_{2} \mathrm{O}_{3}$ 
doped, and Co-doped CdS nanoparticles using hydrothermal and photo-assisted deposition methods. The photo decoloration studies over malachite green have proven that doping $\mathrm{CdS}$ would increase its catalytic performance due to a decrease in the bandgap. While $0.5 \mathrm{wt} \% \mathrm{Pt}$ doped CdS has decolorized malachite green only $80 \%$ in $60 \mathrm{~min}$, the $0.5 \mathrm{wt} \% \mathrm{Pt}, \mathrm{In}_{2} \mathrm{O}_{3}$ codoped CdS reached $100 \%$ same time.

The past three decades have seen extensive work on doping chalcogenide NPs with multiple d-block elements to achieve anticipated changes in electronic, optical, and structural properties. These doped photocatalytic nanomaterials have improved the efficiency of visiblelight-driven photocatalysis. Among other dopants, noble metal dopants exhibit excellent photocatalytic efficiencies. Therefore, $\mathrm{Ag}^{+}, \mathrm{Sn}^{2+}$, and $\mathrm{Pb}^{2+}$ ions were selectively chosen as dopants in CdS NPs. The ecofriendly and economical synthesis of semiconductor photocatalytic materials is a promising route from the environment and industrial applications. A very few reports were available in the literature on the plant extract assisted biosynthesis of CdS NPs.

In this research paper, we have attempted the synthesis of $\mathrm{CdS}$ nanoparticles by a modified co-precipitation technique using $O$. Sanctum leaf extract, which acts both as a green reducer as well as capping agent. It is a safe, non-polluting, and better alternative to conventional chemical methods. These materials were well characterized by using X-ray diffraction, transmission electron microscopy, and UV-Vis spectroscopy techniques. These nanoparticles showed a wide bandgap due to the quantum confinement effect. They showed moderate photocatalytic activity on methylene blue dye under sunlight. To understand the doping effect on photocatalytic efficiency, these nanoparticles were doped with $\mathrm{Ag}^{+}, \mathrm{Sn}^{2+}$, and $\mathrm{Pb}^{2+}$ ions with varying percentages (\% concerning molarity of $\mathrm{Cd}$ ). Upon doping, the resulting $\mathrm{CdS}$ nanoparticles exhibit improved photocatalytic efficiency due to the narrow bandgap. This work will help advance current efforts to improve the photocatalysis of CdS NPs by visible light.

Tulsi (O. Sanctum), the holy herb of the Indian sub-continent, is well known for its medical and health benefits [34]. In Ayurveda, the traditional holistic medical system of India, it is considered as a principal herb. It is utilized in treating eye infections, malaria, diarrhea, arthritis, lung disorders, asthma, bronchitis, etc. It can even inhibit the growth of HIV and carcinogenic cells [35]. It possesses anticancer [36], antibacterial [37], analgesic, adaptogenic, diaphoretic [38], hepatoprotective, cardioprotective actions [39] and boosts the immunity power. The leaf extract of $O$. Sanctum consists of flavonoids, ursolic acid, eugenol, triterpenes, polyphenols, etc. [40,41]. These natural biomolecules possess excellent reducing and capping activities, thus enabling the O. Sanctum leaf extract to synthesize and stabilize nanomaterials via green chemistry technologies.

\section{Materials and Methods}

\subsection{Materials.}

Fresh leaves of Ocimum sanctum were collected from the ANU herbal garden, Acharya Nagarjuna University. The collected leaves were thoroughly cleaned with distilled water and further used for leaf extract preparation. $\mathrm{Cd}\left(\mathrm{CH}_{3} \mathrm{COO}\right)_{2}, \mathrm{AgNO}_{3}, \mathrm{~Pb}\left(\mathrm{NO}_{3}\right)_{2}, \mathrm{SnCl}_{2}$ and $\mathrm{Na}_{2} \mathrm{~S} \bullet 9 \mathrm{H}_{2} \mathrm{O}$ (Merck Ltd., India) were used as received. 


\subsection{General synthesis of CdS nanomaterials.}

The preparation of $O$. sanctum leaf extract was adopted from the literature procedures [42]. Initially, $25 \mathrm{~g}$ of cleaned leaves were boiled in $100 \mathrm{~mL}$ distilled water for $1 \mathrm{~h}$. The cooled reaction contents were filtered through Whatman filter paper to obtain brownish leaf extract and stored at $4^{\circ} \mathrm{C}$ until further usage.

First, $1.33 \mathrm{~g}$ of cadmium acetate was dissolved in O. Sanctum leaf extract ( $35 \mathrm{~mL})$. To this homogeneous solution, sodium sulfide $(0.4 \mathrm{~g}$ of sodium sulfide dissolved in $10 \mathrm{~mL}$ of $\mathrm{O}$. Sanctum leaf extract) solution was added dropwise. A color change from brown to bright yellow was observed during this time, a visual confirmation for $\mathrm{CdS}$ nanoparticles' formation. The resultant solution was stirred for $1 \mathrm{~h}$ and centrifuged, washed with plenty of water and ethanol. The obtained product was dried at $100{ }^{\circ} \mathrm{C}$ to get the final $\mathrm{CdS}$ nanoparticles. Similar procedures were followed for the preparation of metal ion-doped $\mathrm{CdS}$ nanoparticles except for the addition of 5 and $10 \%$ (percentage concerning the concentration of $\mathrm{Cd}^{+2}$ ion) of $\mathrm{SnCl}_{2}$, $\mathrm{Pb}\left(\mathrm{NO}_{3}\right)_{2}$ and $\mathrm{AgNO}_{3}$ in $\mathrm{Cd}\left(\mathrm{CH}_{3} \mathrm{COO}\right)_{2}$ solution. A synthetic schematic of doped and undoped $\mathrm{CdS}$ nanoparticles manufactured using the previously prepared leaf extract was depicted in Fig. 1 .
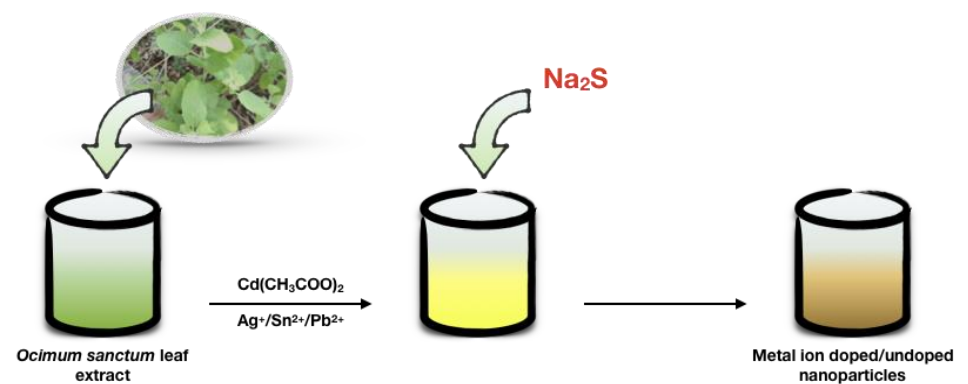

Figure 1. Schematic illustration of the synthesis of doped/undoped CdS nanoparticles using Ocimum sanctum leaf extract.

\subsection{Photocatalytic studies.}

The degradation of methylene blue dye under sunlight was studied using doped and undoped CdS nanoparticles as photocatalysts. All photocatalytic experiments were carried with due precautions under similar conditions on sunny days from 10:30 am to 4:30 pm. Before performing photocatalytic experiments, $100 \mathrm{~mL}$ of dye solution containing $0.1 \mathrm{mg} / \mathrm{mL}$ of synthesized nanoparticles was stirred constantly for $1 \mathrm{~h}$. The stirring was done in a dark environment until adsorption/desorption equilibrium was achieved. A $5 \mathrm{~mL}$ aliquots were sampled at equal intervals. The collected aliquots were analyzed by recording absorption spectra in the range of 500 to $800 \mathrm{~nm}$. The photocatalytic efficiency was calculated by using the formula:

$$
\text { Photocatalytic efficiency }(\%)=\left(\frac{C_{0}-C_{\mathrm{t}}}{C_{0}}\right) \times 100=\left(\frac{A_{0}-A_{\mathrm{t}}}{A_{0}}\right) \times 100
$$

Where, $\mathrm{C}_{0}$ represents the initial concentration of methylene blue and $\mathrm{C}_{\mathrm{t}}$ is the concentration of dye after time t. Further, $A_{0}$ is the absorbance of pure methylene blue before photocatalysis experiments, and $A_{t}$ is the absorbance after exposure to sunlight for time $t$.

\subsection{Instrumental methods.}

Crystallographic analysis of obtained CdS nanoparticles was acquired on Bruker AXS model D8 advanced powder X-ray diffractometer ran with $\mathrm{Cu}-\mathrm{K} \alpha$ radiation $(\lambda=1.54 \AA$ ), and 
the diffraction intensities were measured at $2 \theta$ values from 10 to $100^{\circ}$. The transmission electron microscopy (TEM) images were recorded using FEI Techni $\mathrm{G}^{2}$ S-twin transmission electron microscopy. It was operated at an accelerating voltage of $200 \mathrm{kV}$. The UltravioletVisible (UV-Vis) absorption spectra were recorded on JASCO V-550 (Japan) spectrophotometer between 200 to $800 \mathrm{~nm}$ wavelength range.

\section{Results and Discussion}

\subsection{Structural analysis of doped and undoped nanoparticles.}

The UV-Vis spectral analysis of $5 \%$ and $10 \%$ metal ion-doped and undoped CdS nanoparticles magnificently synthesized via $O$. sanctum leaf extract as a bio capping agent was first utilized to confirm the CdS. Fig. 2(a) shows the absorbance spectra of bio capped CdS nanoparticles with a broad peak at $453 \mathrm{~nm}$. On doping with metal ions, a slight redshift of this peak was observed, signifying the bandgap decrease. At the same time, on increasing the dopant quantity, this shift is prominent, and also a sudden fall in the peak intensity at $230 \mathrm{~nm}$ was observed, indicating the formation of trap states. The UV-Vis absorption at $262 \mathrm{~nm}$ can be ascribed to the biomolecules covered on the nanoparticle's surface. Tauc's plots were plotted from these UV-Vis spectra in order to estimate the bandgap of these materials. Pure CdS nanoparticles exhibit an electronic bandgap of $2.37 \mathrm{eV}$, but on doping with metal ions, this bandgap has reduced, whose values were presented in Table 1. The reduction in the bandgap was further showed an increment with the percentage of doping. For instance, the bandgap of $2.37 \mathrm{eV}$ of pure $\mathrm{CdS}$ NPs was reduced to 2.28 and $2.13 \mathrm{eV}$ on doping with $5 \%$ and $10 \% \mathrm{Ag}$, respectively. A similar trend was observed in the case of $\mathrm{Pb}$ and $\mathrm{Sn}$ doping.
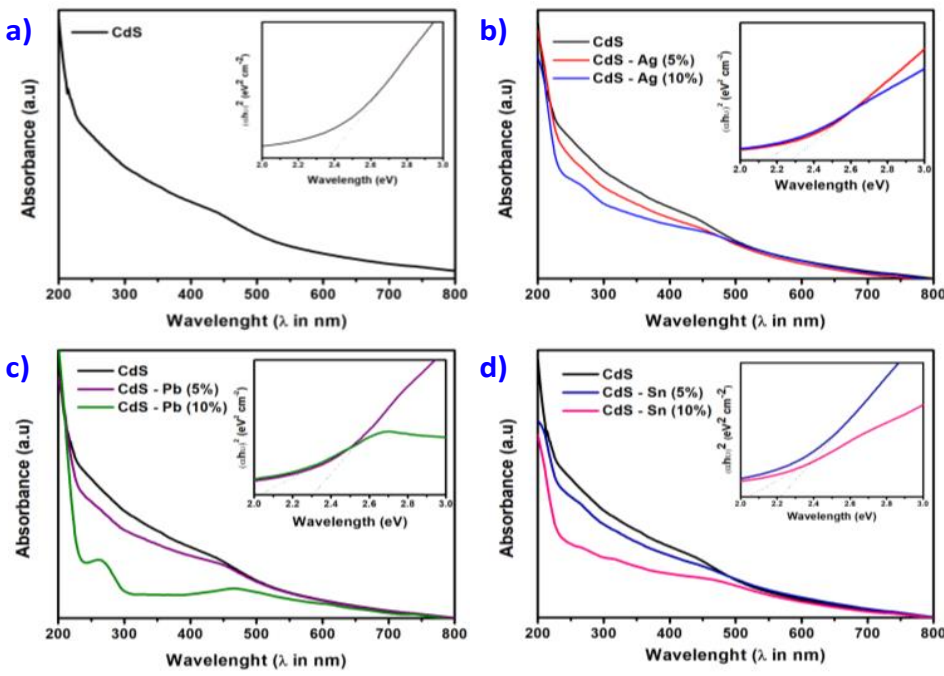

Figure 2. UV-Vis Spectra of O.Sanctum leaf extract capped a) pure, b) pure and Ag-doped, c) pure and $\mathrm{Pb}$ doped, d) pure and Sn doped CdS nanoparticles with insets showing Tauc's plots.

Table 1. The measured band gap values, d-spacing, and crystallite sizes of various doped and undoped CdS nanoparticles.

\begin{tabular}{l|c|c|c|c} 
Sample & Band gap $(\mathbf{e V})$ & $\mathbf{2 \theta}(\mathbf{d e g r e e s})$ & d-spacing $(\mathbf{n m})$ & Crystallite size (D in nm) \\
\hline $\mathbf{C d S}$ & 2.37 & 28.23 & 0.315 & 1.72 \\
\hline $\mathbf{C d S}-\mathbf{P b}(\mathbf{5 \%})$ & 2.30 & 28.17 & 0.316 & 1.60 \\
\hline $\mathbf{C d S}-\mathbf{P b}(\mathbf{1 0 \%})$ & 2.03 & 27.46 & 0.324 & 1.78 \\
\hline $\mathbf{C d S}-\mathbf{A g}(\mathbf{5 \%})$ & 2.28 & 28.04 & 0.317 & 2.14 \\
\hline $\mathbf{C d S}-\mathbf{A g}(\mathbf{1 0 \%})$ & 2.13 & 27.38 & 0.325 & 1.91 \\
\hline $\mathbf{C d S}-\mathbf{S n} \mathbf{5 \%})$ & 2.22 & 28.32 & 0.314 & 1.76 \\
\hline $\mathbf{C d S}-\mathbf{S n}(\mathbf{1 0 \%})$ & 2.04 & 27.55 & 0.323 & 1.65
\end{tabular}


Structural properties of green synthesized nanoparticles were then characterized using XRD patterns, as shown in Fig. 3. The X-ray diffractograms of pure CdS nanoparticles synthesized without leaf extract showed peaks at $2 \theta=27,44$, and $52^{\circ}$ corresponding to (111), (220) and (311) crystal planes of CdS pertaining to the cubic structure with JCPDS No.652887. In the presence of leaf extract, these peaks have broadened, indicating the decreased size of formed particles by the effective capping power of biomolecules existing in the leaf extract. It is observed that with doping $\mathrm{Pb}^{2+}, \mathrm{Ag}^{+}$and $\mathrm{Sn}^{2+}$, that there was no key difference in the XRD patterns; nonetheless, there was a slight left shift on the increment of doping amount indicating the incorporation of metal ions in lattice planes of $\mathrm{CdS}$. The crystallite sizes tabulated in table 1 were measured with Debye - Scherrer formulas as given below.

$$
D=\frac{0.9 \lambda}{\beta \cos \theta}
$$

Where, $\beta$ is the full width at half maximum of peak belonging to (111) plane, $\theta$ is the incident angle, and $\lambda$ is the wavelength of $\mathrm{X}$-ray.
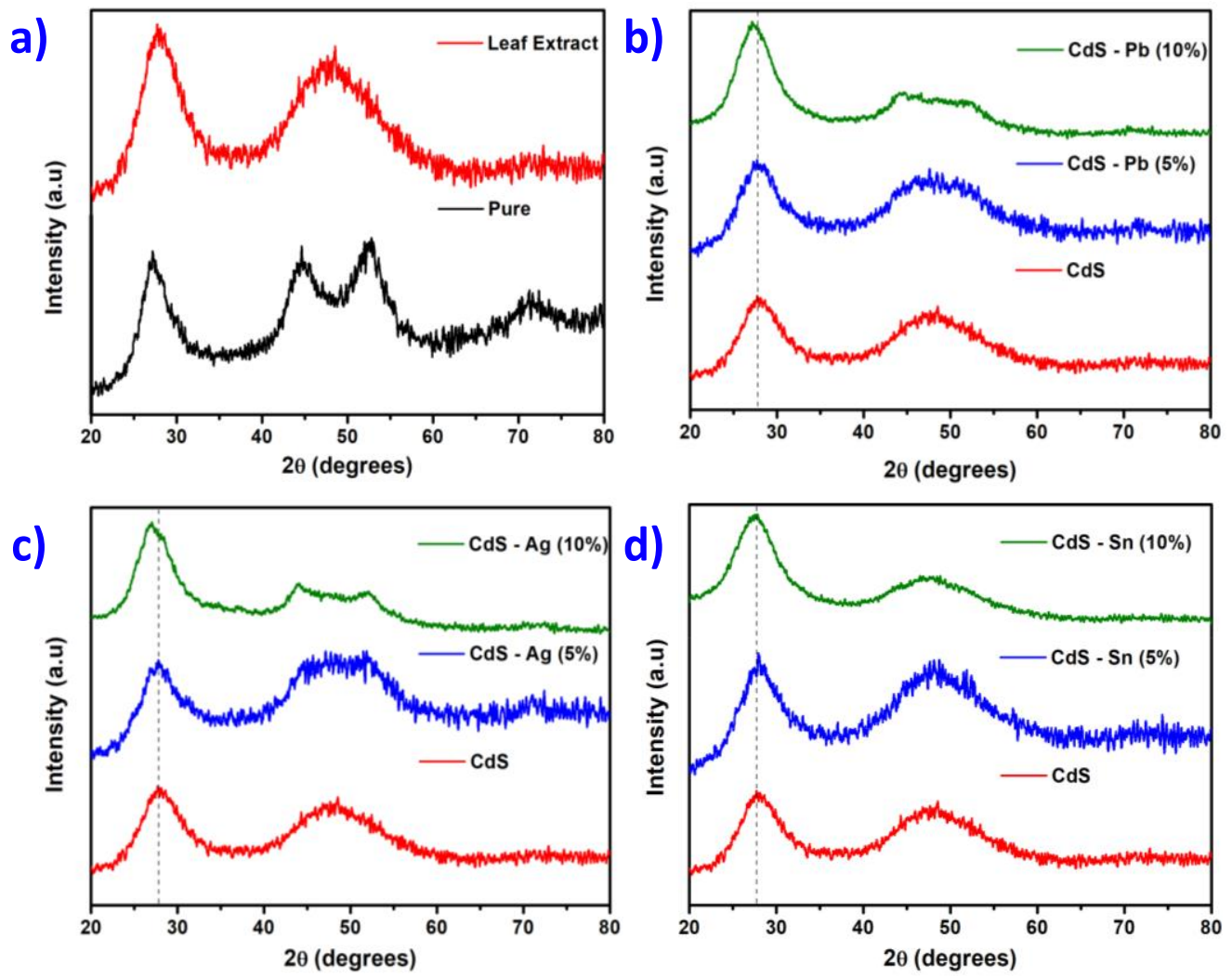

Figure 3. XRD patters of a) CdS nanoparticles synthesized with and without leaf extract, b) $\mathrm{Pb}, \mathrm{c}$ ) $\mathrm{Ag}$, d) $\mathrm{Sn}$ doped CdS nanoparticles.

Transmission electron microscopy images of $\mathrm{CdS}$ nanoparticles along with $\mathrm{Ag}, \mathrm{Sn}$, and $\mathrm{Pb}$ dopants were shown in Fig. 4. TEM images revealed that the particle sizes were $\sim 6 \mathrm{~nm}$. HR-TEM images of these nanoparticles do not exhibit any considerable changes in the dspacing when doped with different metals, which was consistent with those obtained from XRD. Further, the HR-TEM images showed CdS nanoparticles' clusters; the aggregation into small clusters could be attributed to the strong intermolecular forces that operate between these CdS nanoparticles. 

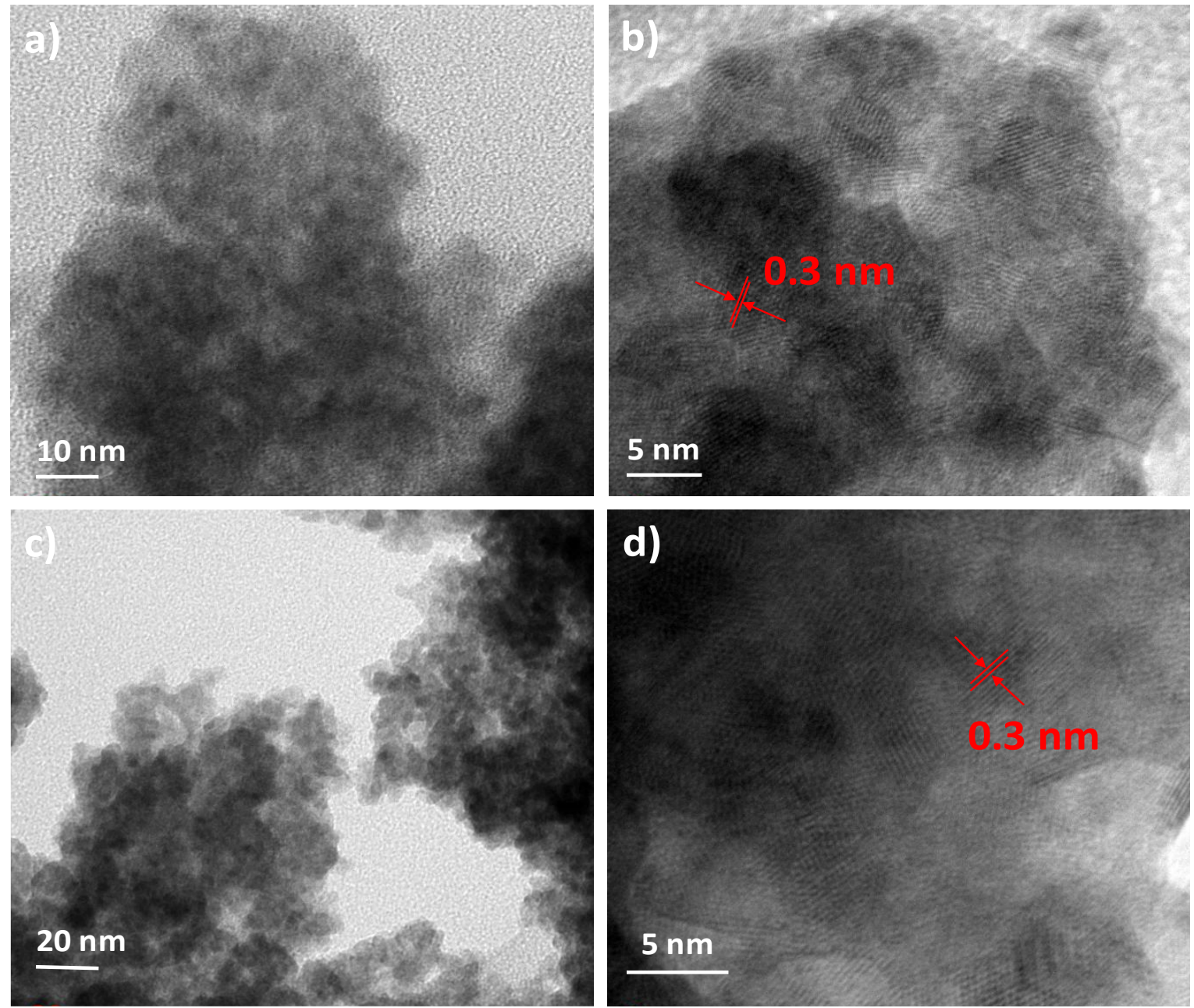

\section{d)}
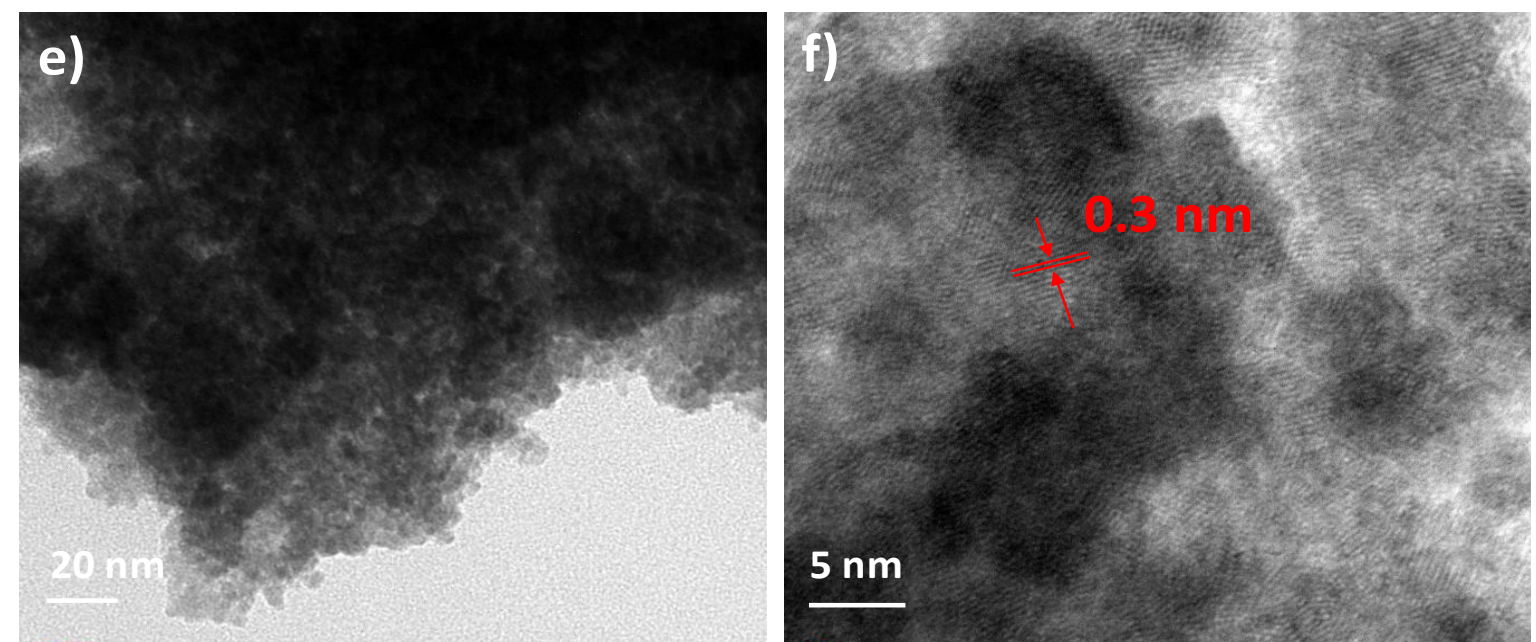

Figure 4. High-resolution transmission electron microscope images of CdS nanoparticles doped with $10 \%(\mathrm{a}, \mathrm{b})$ $\mathrm{Ag}$ (c, d) $\mathrm{Sn}(\mathrm{e}, \mathrm{f}) \mathrm{Pb}$ doped CdS nanoparticles.

\subsection{Dye degradation studies.}

To understand the influence of doping over photocatalytic ability, these doped and undoped CdS nanoparticles were tested for photodegradation of methylene blue dye in the presence of sunlight. Prior to this, the doped and undoped CdS nanoparticles were distributed in the dye solution and constantly agitated for $1 \mathrm{~h}$ in the dark in order to attain the adsorption/desorption equilibrium of dye with the surface of the catalyst. Fig. 5 depicts the 
temporal absorption spectra of methylene blue taken at regular intervals during photocatalysis experiments.

a)

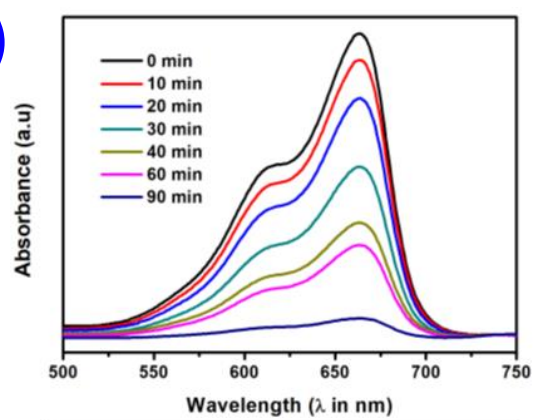

c)

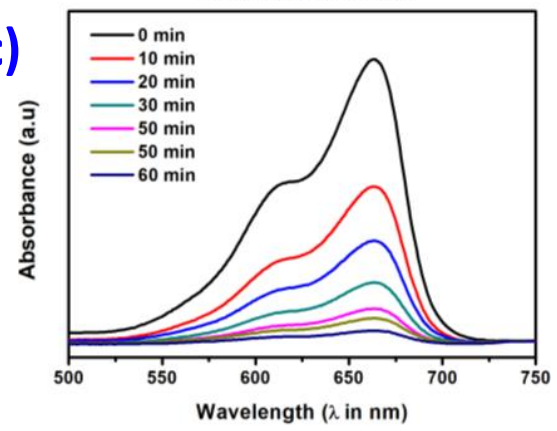

e)

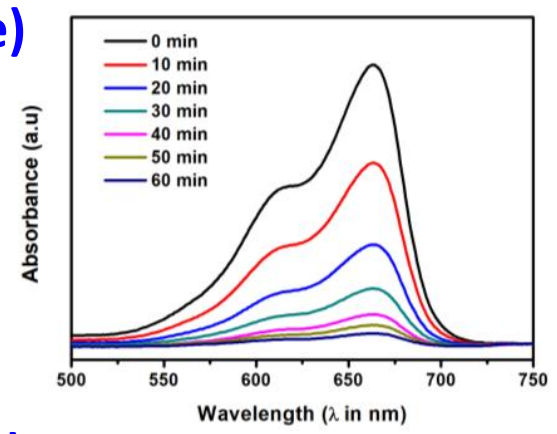

g)
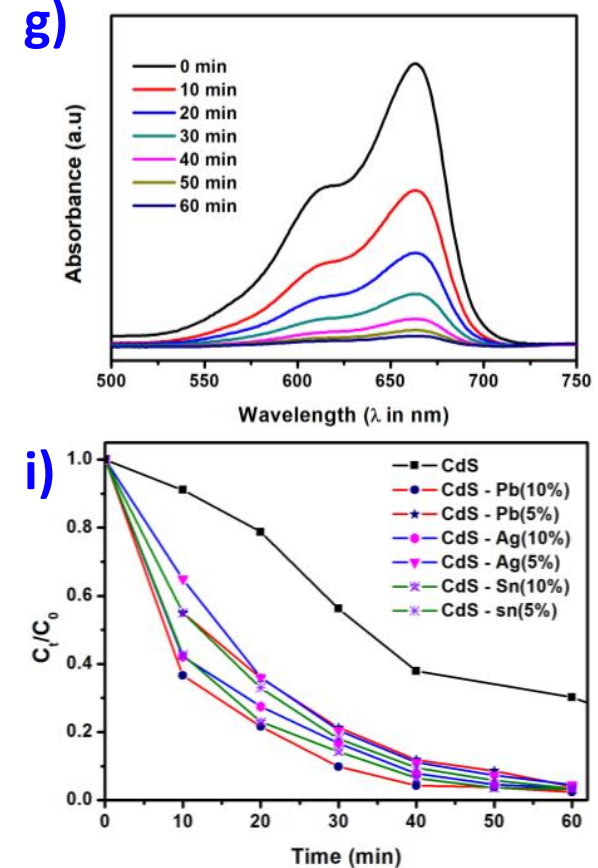

b)
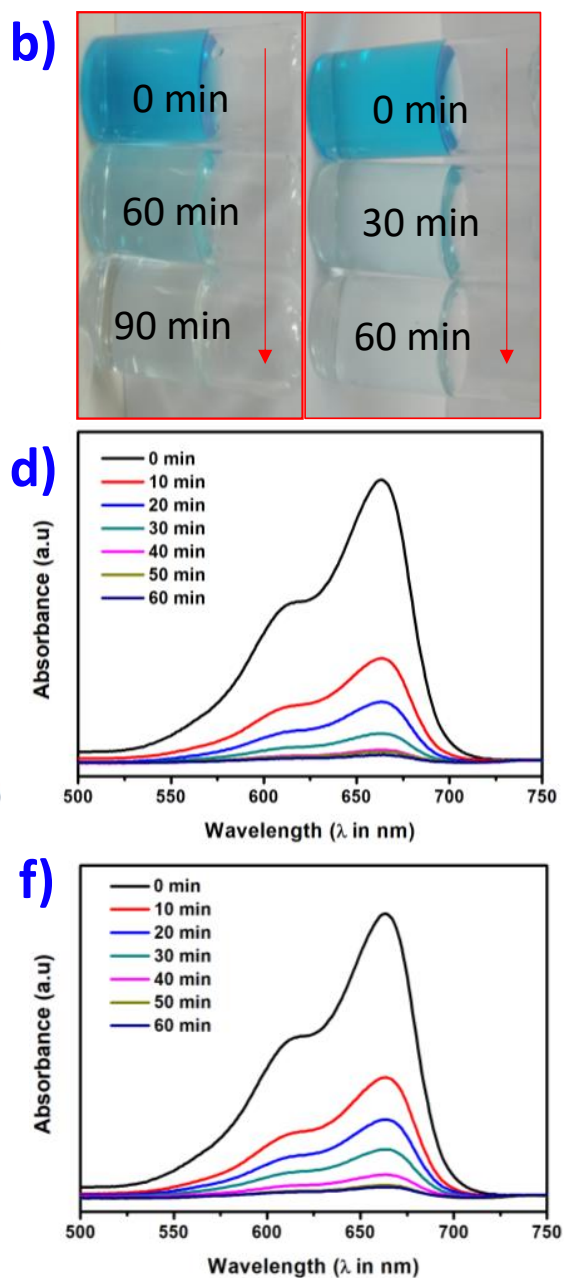

h)
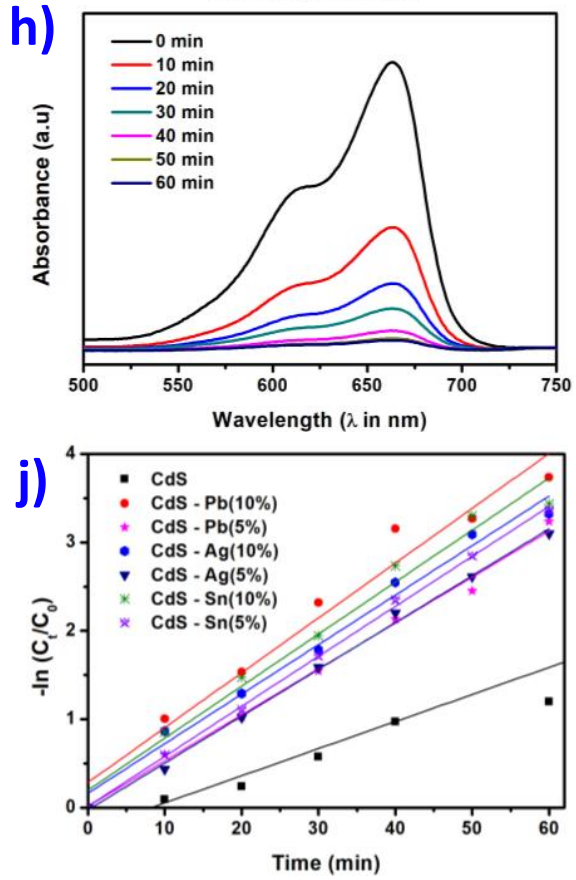

Figure 5. Absorption spectral data presenting photocatalytic degradation of methylene blue by catalysis of a) pure $\mathrm{CdS}$, c) $5 \% \mathrm{~Pb}$, d) $10 \% \mathrm{~Pb}$, e) $5 \% \mathrm{Ag}$, f) $10 \% \mathrm{Ag} \mathrm{g}$ ) $5 \% \mathrm{~Pb}$, h) $10 \% \mathrm{~Pb}$ doped CdS nanoparticles. i) degradation activity, $\mathrm{j}$ ) the kinetic data of degradation of methylene blue overdoped and undoped $\mathrm{CdS}$ nanoparticles. $\mathrm{b}$ ) Pictographs of methylene blue solutions taken during photocatalytic experiments in the that contain undoped and $10 \% \mathrm{~Pb}$ doped $\mathrm{CdS}$ nanoparticles. 
In all the cases, it was observed that the concentration of dye had reduced gradually, and the absorption peak corresponding to methylene blue has almost disappeared after specific timings. However, this process was not the same for all the samples. From the photodecoloration activity of different samples shown in Fig. 5(i), it was clear that the green synthesized CdS nanoparticles showed profound improvement in photodecoloration on doping with different metal ions. Also, this enhancement was more on increasing the amounts of metal ions that were doped. This infers that the insertion of metal ions in the $\mathrm{CdS}$ crystal lattice would enhance the catalytic activity. Bare O. sanctum leaf extract assisted synthesized CdS nanoparticles showed a photodegradation efficiency of only $94.04 \%$ for $1.5 \mathrm{~h}$. However, on doping with $5 \%$ of $\mathrm{Ag}^{+}, \mathrm{Pb}^{2+}, \mathrm{Sn}^{2+}$ ions, this efficiency has increased to 95.50, 95.94, 96.49 $\%$, respectively, in $1 \mathrm{~h}$ of photocatalysis process. On further increasing the amounts of doping, there was a drastic improvement in degradation efficiency, reaching 60\% within 10 min. Figure 5(j) understood that the photodegradation kinetics follows a pseudo-first-order pathway, demonstrated by the following equation.

$$
-\ln \left(\mathrm{C}_{\mathrm{t}} / \mathrm{C}_{\mathrm{o}}\right)=\mathrm{kt}
$$

where $k$ is the kinetic constant, which was observed to be enhanced on doping. Kinetic constants calculated by the above formula, regression coefficients, and photo degeneration efficiency of given catalysts over methylene blue dye were detailed in Table 2.

Table 2. Kinetic constant $(K)$, Regression coefficient $\left(\mathrm{R}^{2}\right)$, and photocatalytic efficiency values calculated for 1 $\mathrm{h}$ of the photocatalytic process in the presence of various $\mathrm{CdS}$ nanoparticles.

\begin{tabular}{l|c|c|c} 
Catalyst & $\boldsymbol{K}\left(\mathbf{m i n}^{-1}\right)$ & $\mathbf{R}^{\mathbf{2}}$ & Degradation Efficiency $(\boldsymbol{\%})$ \\
\hline $\mathbf{C d S}$ & 0.0306 & 0.929 & 69.78 \\
\hline $\mathbf{5 \%} \mathbf{P b}-\mathbf{C d S}$ & 0.0518 & 0.993 & 95.94 \\
\hline $\mathbf{1 0} \% \mathbf{P b}-\mathbf{C d S}$ & 0.062 & 0.961 & 98.01 \\
\hline $\mathbf{5 \%} \mathbf{A g}-\mathbf{C d S}$ & 0.0529 & 0.996 & 95.50 \\
\hline $\mathbf{1 0} \% \mathbf{A g}-\mathbf{C d S}$ & 0.0559 & 0.982 & 96.78 \\
\hline $\mathbf{5 \%} \mathbf{S n}-\mathbf{C d S}$ & 0.0565 & 0.998 & 96.49 \\
\hline $\mathbf{1 0} \% \mathbf{S n}-\mathbf{C d S}$ & 0.0588 & 0.976 & 97.76
\end{tabular}

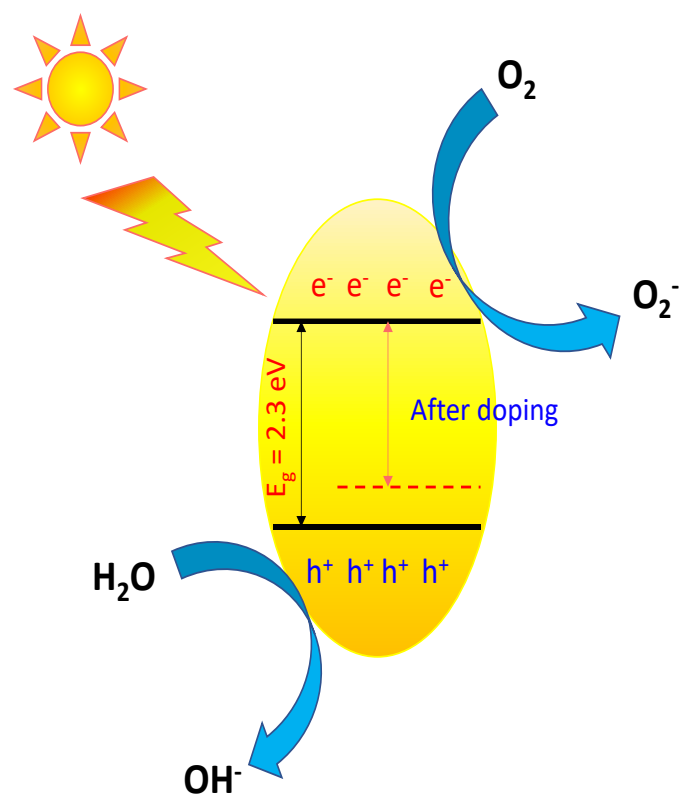

Figure 6. Schematic presentation of dye degradation mechanism for pure and doped CdS photocatalyst.

Based on the above experimental results, the probable mechanism for the enhanced photocatalytic activities of overdoped CdS nanoparticles was depicted in Fig. 6 and can be 
explained as follows. On partial entry of $\mathrm{Pb}, \mathrm{Sn}$, and/or $\mathrm{Ag}$ into the lattice of $\mathrm{CdS}$, they create an electron donor level in the forbidden bandgap of pure $\mathrm{CdS}$. This resulted in the reduction of bandgap, which was highly favorable not only for the harvesting of sunlight but also for the generation of electron-hole pairs $[43,44]$. At the same time, it was estimated that these dopant metal ions could act as trapping sites/sinks of photoinduced electrons, thus reducing the chances of electron-hole recombination, which was the major concern to be addressed in photocatalysis. Electron present in the conduction band reaches the catalyst's surface, and this electron can reduce molecular oxygen to superoxide anion. This will further react with water to form $\mathrm{H}_{2} \mathrm{O}_{2}$ and finally forming $\mathrm{OH}^{\bullet}$ radicals which degrade the organic dye adsorbed on the catalyst $[7,45,46]$. The holes present in the valance band can oxidize the adsorbed dye forming a reactive intermediate and ultimately destroy the dye to simple biodegradable molecules.

Table 3. Comparison of the dye degradation efficiencies reported with our results.

\begin{tabular}{l|l|l|l|l|l} 
S No & Catalyst preparation Method & Catalyst & Dye & Degradation Efficiency (\%) & Ref \\
\hline 1 & $\begin{array}{l}\text { Sonochemical green co- } \\
\text { precipitation }\end{array}$ & $\mathrm{CdS}$ & $\mathrm{MB}$ & 85.53 \\
\hline 2 & Co-precipitation & $\mathrm{Zn}_{1-\mathrm{x}} \mathrm{Cu}_{\mathrm{x}} \mathrm{S}$ & $\mathrm{MB}$ & 65 & 36 \\
\hline 3 & Co-precipitation & $\mathrm{Zn}_{1-\mathrm{x}} \mathrm{Mn}_{\mathrm{x}} \mathrm{S}$ & $\mathrm{MB}$ & 50 & 18 \\
\hline 4 & Co-precipitation, reflux & $\mathrm{Cd}_{1-\mathrm{x}} \mathrm{M} \mathrm{S}$ & $\mathrm{MB}$ & 82 & 18 \\
\hline 5 & Chemical precipitation & $\mathrm{Cd}_{1-\mathrm{x}} \mathrm{Fe}$ & $\mathrm{MB}$ & $100 \%$ but 3-5 h \\
\hline 6 & Hydrothermal & $\mathrm{Ni} / \mathrm{Ce}$ doped CdS & $\mathrm{RB}$ & 90 & 19 \\
\hline 7 & Green co-precipitation & $10 \% \mathrm{Ag}-\mathrm{CdS}$ & $\mathrm{MB}$ & 96.7 & 22 \\
\hline 8 & Green co-precipitation & $10 \% \mathrm{Sn}-\mathrm{CdS}$ & $\mathrm{MB}$ & 97.7 & 37 \\
\hline 9 & Green co-precipitation & $10 \% \mathrm{~Pb}-\mathrm{CdS}$ & $\mathrm{MB}$ & 98 & Present work \\
\hline
\end{tabular}

We have compared the dye degradation efficiencies with previously reported results in Table 3. From table 3, it is clear that our catalysts showed improved dye degradation efficiencies than the literature that reported similar chalcogenide photocatalysts.

\section{Conclusions}

To conclude, undoped and $\mathrm{Ag}^{+}, \mathrm{Sn}^{2+}, \mathrm{Pb}^{2+}$ ions doped $\mathrm{CdS}$ nanoparticles were successfully manufactured using Ocimum sanctum leaf extract as a capping and reducing agent. The present method utilized the biomolecules present in the leaf extract as capping agents, thus reducing hazardous and costly reagents in the synthetic process. The obtained nanoparticles were characterized by using UV-Vis spectroscopy, XRD, and TEM techniques. UV-Vis spectra revealed the change in bandgap on doping with various metal ions. XRD and TEM also confirmed the change in d-spacing, which signifies the occupancy of dopants in the CdS crystal lattice. The as-synthesized nanoparticles were employed in photocatalytic degradation of Methylene Blue dye under sunlight. The doped CdS nanoparticles showed better photocatalytic efficiencies than the pure $\mathrm{CdS}$ nanoparticles. Among them, $\mathrm{Pb}$ doped $\mathrm{CdS}$ nanoparticles showed superior photocatalytic efficiency over Sn and Ag-doped CdS nanoparticles. The photocatalytic efficacies of metal-doped cadmium sulfide nanoparticles were in the order $(\mathrm{Pb}$ $>\mathrm{Sn}>\mathrm{Ag})$.

\section{Funding}

This research received no external funding.

\section{Acknowledgments}

This research has no acknowledgment. 


\section{Conflicts of Interest}

\section{The authors declare no conflict of interest.}

\section{References}

1. Iravani, S. Green synthesis of metal nanoparticles using plants. Green Chemistry 2011, 13, 2638-2650, https://doi.org/10.1039/C1GC15386B.

2. Sharma, D.; Kanchi, S; Bisetty, K. Biogenic synthesis of nanoparticles: A review. Arabian journal of chemistry 2019, 12, 3576-3600, https://doi.org/10.1016/j.arabjc.2015.11.002.

3. Gahlawat, G.; Choudhury, A.R. A review on the biosynthesis of metal and metal salt nanoparticles by microbes. RSC advances 2019, 9, 12944-12967, https://doi.org/10.1039/C8RA10483B.

4. Heikal Y.M.; Abdel-Aziz H.M.M. Biogenic Nanomaterials and Their Applications in Agriculture. In: Biogenic Nano-Particles and their Use in Agro-ecosystems. Ghorbanpour, M.; Bhargava, P.; Varma, A.; Choudhary, D. (eds) Springer, Singapore. 2020; pp. 489-414, https://doi.org/10.1007/978-981-15-2985$6 \_25$.

5. Duan, H.; Wang, D.; Li, Y. Green chemistry for nanoparticle synthesis. Chemical Society Reviews 2015, 44, 5778-5792, https://doi.org/10.1039/C4CS00363B

6. de Souza, T.A.J.; Souza, L.R.R.; Franchi, L.P. Silver nanoparticles: An integrated view of green synthesis methods, transformation in the environment, and toxicity. Ecotoxicology and environmental safety 2019, 171, 691-700, https://doi.org/10.1016/j.ecoenv.2018.12.095.

7. Mosali, V.S.S.; Qasim, M.; Mullamuri, B.; Chandu, B.; Das, D. Synthesis and characterization of $\mathrm{Ag} / \mathrm{CoFe} 2 \mathrm{O} 4 /$ polyaniline nanocomposite for photocatalytic application. Journal of Nanoscience and Nanotechnology, 2017, 17, 8918-8924, https://doi.org/10.1166/jnn.2017.13903.

8. Al-Ghouti, M.A.; Khraisheh, M.A.M.; Allen, S.J.; Ahmad, M.N. The removal of dyes from textile wastewater: a study of the physical characteristics and adsorption mechanisms of diatomaceous earth. Journal of environmental management 2003, 69, 229-238, https://doi.org/10.1016/j.jenvman.2003.09.005.

9. Dos Santos, A.B.; Cervantes, F.J.; van Lier, J.B. Review paper on current technologies for decolourisation of textile wastewaters: perspectives for anaerobic biotechnology. Bioresource technology 2007, 98, 23692385, https://doi.org/10.1016/j.biortech.2006.11.013.

10. Liu, W.; Yuan, K.; Liu, P.; Chen, M. Construction of detachable core/shell Fe3O4@ C supported noble metal catalysts and their catalytic performance. Colloids and Surfaces A: Physicochemical and Engineering Aspects 2019, 580, https://doi.org/10.1016/j.colsurfa.2019.123729.

11. Abharya, A.; Gholizadeh, A. Structural, optical and magnetic feature of core-shell nanostructured Fe3O4@ GO in photocatalytic activity. Iranian Journal of Chemistry and Chemical Engineering (IJCCE) 2020, 39, 49-58, https://doi.org/10.30492/IJCCE.2020.34296.

12. Zhang, J.; Li, L.; Shi, R.; Mei, J.; Xiao, Z.; Ma, W. An efficient approach for the synthesis of magnetic separable Fe3O4@ TiO2 core-shell nanocomposites and its magnetic and photocatalytic performances. Materials Research Express 2019, 6, https://doi.org/10.1088/2053-1591/ab3531.

13. Bokare, A.D.; Chikate, R.C.; Rode, C.V.; Paknikar, K.M. Iron-nickel bimetallic nanoparticles for reductive degradation of azo dye Orange $\mathrm{G}$ in aqueous solution. Applied Catalysis B: Environmental 2008, 79, 270278, https://doi.org/10.1016/j.apcatb.2007.10.033.

14. Joseph, S.; Mathew, B. Microwave-assisted green synthesis of silver nanoparticles and the study on catalytic activity in the degradation of dyes. Journal of Molecular Liquids 2015, 204, 184-191, https://doi.org/10.1016/j.molliq.2015.01.027.

15. Sankar, R.; Manikandan, P.; Malarvizhi, V.; Fathima, T.; Shivashangari, K.S.; Ravikumar, V. Green synthesis of colloidal copper oxide nanoparticles using Carica papaya and its application in photocatalytic dye degradation. Spectrochimica Acta Part A: Molecular and Biomolecular Spectroscopy 2014, 121, 746750, https://doi.org/10.1016/j.saa.2013.12.020.

16. Ayodhya, D.; Venkatesham, M.; Kumari, A.S.; Reddy, G.B.; Ramakrishna, D.; Veerabhadram, G. Synthesis, characterization, fluorescence, photocatalytic and antibacterial activity of CdS nanoparticles using Schiff base. Journal of fluorescence 2015, 25, 1481-1492, https://doi.org/10.1007/s10895-015-1639-5.

17. Hernández-Gordillo, A.; Ramos, A.B.; Acevedo-Peña, P.; Jagdale, P.; Tagliaferro, A.; Rodil, S.E. Dependence of the photoactivity of CdS prepared in butanol-ethylenediamine mixture in function of different sacrificial electron donors. Catalysis Today 2020, 341, 59-70, https://doi.org/10.1016/j.cattod.2018.10.057.

18. Davis, A.P.; Hsieh, Y.H.; Huang, C.P. Photo-oxidative dissolution of CdS (s): The effect of complexing agents. Chemosphere 1995, 31, 3093-3104, https://doi.org/10.1016/0045-6535(95)00168-8.

19. Ai, Z.; Zhao, G.; Zhong, Y.; Shao, Y.; Huang, B.; Wu, Y.; Hao, X. Phase junction CdS: High efficient and stable photocatalyst for hydrogen generation. Applied Catalysis B: Environmental 2018, 221, 179-186, https://doi.org/10.1016/j.apcatb.2017.09.002.

20. Xiong, J.; Du, X.; Cheng, G.; Yang, H.; Chen, J.; Dou, S.; Li, Z. One dimensional hierarchical nanostructures composed of $\mathrm{CdS}$ nanosheets/nanoparticles and $\mathrm{Ag}$ nanowires with promoted photocatalytic performance. Inorganic Chemistry Frontiers, 2018, 5, 903-915, https://doi.org/10.1039/C8QI00014J. 
21. Jeevanantham, N; Balasundaram, O.N. Role of Sm dopant on structural, optical and efficient visible light photocatalytic performance of CdS nanoparticles by using simple wet chemical route. Journal of Materials Science: Materials in Electronics 2018, 29, 14073-14083, https://doi.org/10.1007/s10854-018-9539-5.

22. Lilhare, D.; Sinha, T.; Khare, A. Influence of Cu doping on optical properties of (Cd-Zn) S nanocrystalline thin films: a review. Journal of Materials Science: Materials in Electronics 2018, 29, 688-713, https://doi.org/10.1007/s10854-017-7963-6.

23. Ranjith, R.; Krishnakumar, V.; Boobas, S.; Venkatesan, J.; Jayaprakash, J. An Efficient Photocatalytic and Antibacterial Performance of $\mathrm{Ni} / \mathrm{Ce}-\mathrm{Co}$-doped $\mathrm{CdS}$ Nanostructure under Visible Light Irradiation. ChemistrySelect 2018, 3, 9259-9267.

24. Hu, L.; Deng, G.; Lu, W.; Pang, S.; Hu, X. Deposition of CdS nanoparticles on MIL-53 (Fe) metal-organic framework with enhanced photocatalytic degradation of RhB under visible light irradiation. Applied Surface Science 2017, 410, 401-413, https://doi.org/10.1016/j.apsusc.2017.03.140.

25. Sernelius, B.E.; Berggren, K.F.; Jin, Z.C.; Hamberg, I.; Granqvist, C.G. Band-gap tailoring of ZnO by means of heavy Al doping. Physical Review B 1988, 37, https://doi.org/10.1103/PhysRevB.37.10244.

26. Yang, X.; Jia, Z.; Cheng, X.; Luo, N.; Choi, M.M. Synthesis of N-acetyl-1-cysteine capped Mn: doped CdS quantum dots for quantitative detection of copper ions. Spectrochimica Acta Part A: Molecular and Biomolecular Spectroscopy 2018, 199, 455-461, https://doi.org/10.1016/j.saa.2018.04.003.

27. Kanwal, Z.; Usmani, M.N. Shift in optical properties of Mn doped CdS (A DFT+ U study). Materials Research Express 2018, 5, https://doi.org/10.1088/2053-1591/aaa80b.

28. Pouretedal, H.R.; Norozi, A.; Keshavarz, M.H.; Semnani, A. Nanoparticles of zinc sulfide doped with manganese, nickel and copper as nanophotocatalyst in the degradation of organic dyes. Journal of Hazardous Materials 2009, 162, 674-681, https://doi.org/10.1016/j.jhazmat.2008.05.128.

29. Pouretedal, H.R.; Eskandari, H.; Keshavarz, M.H.; Semnani, A. Photodegradation of Organic Dyes using Nanoparticles of Cadmium Sulfide Doped with Manganese, Nickel and Copper as Nanophotocatalyst. Acta Chimica Slovenica 2009, 56, 353-361.

30. Kaur, J.; Sharma, M.; Pandey, O.P. Photoluminescence and photocatalytic studies of metal ions (Mn and Ni) doped ZnS nanoparticles. Optical Materials, 2015, 47, 7-17, https://doi.org/10.1016/j.optmat.2015.06.022.

31. Rajabi, H.R.; Farsi, M. Effect of transition metal ion doping on the photocatalytic activity of ZnS quantum dots: synthesis, characterization, and application for dye decolorization. Journal of Molecular Catalysis A: Chemical 2015, 399, 53-61, https://doi.org/10.1016/j.molcata.2015.01.029.

32. Chauhan, R.; Kumar, A.; Chaudhary, R.P. Visible-light photocatalytic degradation of methylene blue with Fe doped CdS nanoparticles. Applied surface science 2013, 270, 655-660, https://doi.org/10.1016/j.apsusc.2013.01.110.

33. Qusti, A.H.; Malkhasian, A.Y.; Salam, M.A. Enhancement of CdS nanoparticles photocatalytic activity by $\mathrm{Pt}$ and In2O3 doping for the degradation of malachite green dye in water. Journal of Molecular Liquids 2018, 255, 364-369, https://doi.org/10.1016/j.molliq.2018.01.127.

34. Rath, S.K.; Swain, C.S.; Panda, S.M. Tulasi (Ocium Santum) in Dentistry-An Overlook. International Journal of Ayurveda and Pharma Research 2017, 5, 86-88.

35. Verma, S. Chemical constituents and pharmacological action of Ocimum sanctum (Indian holy basil-Tulsi). The Journal of Phytopharmacology 2016, 5, 205-207.

36. Kumar, A.; Rahal, A.; Chakraborty, S.; Tiwari, R.; Latheef, S.K.; Dhama, K. Ocimum sanctum (Tulsi): a miracle herb and boon to medical science-A Review. International Journal of Agronomy and Plant Production, 2013, 4, 1580-9.

37. Rathod, G.P.; Kotecha, B.M.; Sharma, R.; Amin, H.; Prajapati, P.K. In vitro Antibacterial study of two commonly used medicinal plants in Ayurveda: Neem (Azadirachta indica L.) and Tulsi (Ocimum sanctum L.). International Journal of Pharmaceutical \& Biological Archives 2012, 3, 582-586.

38. Singh, V.; Amdekar, S.; Verma, O. Ocimum Sanctum (tulsi): Bio-pharmacological Activities. Webmedcentral Pharmacology 2010, 1, https://doi.org/10.9754/journal.wmc.2010.001046P.

39. Prakash, P.; Gupta, N. Therapeutic uses of Ocimum sanctum Linn (Tulsi) with a note on eugenol and its pharmacological actions: a short review. Indian Journal of Physiology and Pharmacology 2005, 49, 125131.

40. Reshma, K.; Ashalatha, V.R.; Dinesh, M.; Vasudevan, D.M.. Effect of ocimum flavonoids as a radioprotector on the erythrocyte antioxidants in oral cancer. Indian Journal of Clinical Biochemistry 2005 , 20, 160-164, https://doi.org/10.1007/BF02893064.

41. Suanarunsawat, T.; Anantasomboon, G.; Piewbang, C. Anti-diabetic and anti-oxidative activity of fixed oil extracted from Ocimum sanctum L. leaves in diabetic rats. Experimental and therapeutic medicine 2016, 11, 832-840, https://doi.org/10.3892/etm.2016.2991.

42. Mahata, S.; Sahu, A.; Shukla, P.; Rai, A.; Singh, M.; Rai, V.K. The novel and efficient reduction of graphene oxide using Ocimum sanctum L. leaf extract as an alternative renewable bio-resource. New Journal of Chemistry, 2018, 42, 19945-19952, https://doi.org/10.1039/C8NJ04086A.

43. Chandu, B.; Nurbasha, S.; Bollikolla, H.B. A Facile Green Reduction for Graphene-Silver Nanocomposite Using Betel Leaf Extract for the Photocatalytic Degradation of Water Pollutants. ChemistrySelect 2017, 2, 11172-11176. 
44. Chandu, B.; Kurmarayuni, C.M.; Kurapati, S.; Bollikolla, H.B. Green and economical synthesis of graphene-silver nanocomposite exhibiting excellent photocatalytic efficiency. Carbon Letters 2020, 30, 225-233, https://doi.org/10.1007/s42823-019-00091-3.

45. Srinivasan, V.; Hiragond, C.B.; Khanna, P.K.; More, P.V. Surface Engineering of CdS Quantum Dots for Photocatalytic Applications under Direct Sunlight. ChemistrySelect 2018, 3, 8491-8500, https://doi.org/10.1002/slct.201800830.

46. Luo, W.; Hu, F.; Hu, Y.; Dai, H.; Xu, L.; Xu, G.; Jian, Y.; Peng, X. Persulfate enhanced visible light photocatalytic degradation of organic pollutants by construct magnetic hybrid heterostructure. Journal of Alloys and Compounds, 2019, 806, 1207-1219, https://doi.org/10.1016/j.jallcom.2019.07.329. 